\title{
Motion Correction For 3D Volumetric Reconstruction from 2D Multi-Slice Stacks in Fetal MRI
}

\author{
Bin Chen*, PhD \\ Department of Electrical and Computer Engineering, Purdue University Fort Wayne, USA
}

*Corresponding author: Bin Chen, Department of Electrical and Computer

Engineering, Purdue University Fort Wayne, USA

Received Date: October 19, 2020

Published Date: November 05, 2020

\section{Opinion}

Anomalies in brain development may lead to neurological disorders and developmental impairment and may even affect the survival in the prenatal period or later in the early stages of life. Prenatal ultrasonography is the most widely used imaging technique for prenatal care. However, it has limitations to detect abnormalities in brain development due to fetal position, gestational age or skull ossification. Magnetic Resonance Imaging (MRI) has been increasingly used in clinic to quantitatively assess brain growth and development during the fetal and neonatal period with millimeter-level image resolution, high soft tissue contrast and volumetric coverage. It becomes an essential and ideal technique in clinical care to evaluate fetuses who have risks of developmental abnormalities but not apparently shown on prenatal sonogram.

MRI by nature is a relatively slow technique. The detailed volumetric data usually take several minutes to collect, making it susceptible to motion artifacts. The motion in fetal MRI caused by fetal and maternal movement is intermittent and largely unpredictable and uncontrollable which corrupts the spatial encoding in 3D MRI imaging. Therefore, fast 2D slice acquisition followed by 3D reconstruction from stacks of 2D slices becomes the choice in fetal MRI. Each fetal MRI slice is acquired by rapid single shot imaging sequences, such as Echo Planar Imaging (EPI) or single shot fast spin echo (ssFSE), to "freeze" fetal and maternal motion. While each individual slice generally immunes to motion artifact, the whole volume, consisting of multiple 2D motion- free slices, still needs several minutes to acquire. The motion during multi-slice acquisition leads to misalignment in 3D volume reconstruction, which hampers image interpretation and clinical diagnosis. As one of the biggest challenges in fetal MRI, motion correction has been studied from patient motion prevention, MRI pulse sequence development and post processing. This article is to review techniques developed in the past 15 years in fetal MRI motion correction and volume reconstruction from 2D slice stacks.

The first influential method, Snapshot magnetic resonance imaging with Volume Reconstruction (SVR), was proposed by Jiang et al. [1]. SVR uses dynamic single shot scanning to sample the segmented brain region with multiple overlapping slices. Repeated acquisition of slice planes increases the sampling density and ensures high resolution reconstruction under motion. An optimal estimate of a 3D fetal brain is reconstructed with a scattered interpolation approach under the rigid body assumption. Numerous modifications and improvements have been developed based on SVR. One of the improvements was developed by Keraudren et al. [2] by combining fetal brain segmentation with SVR to make the whole registration process fully automatic.

Kim et al. [3] proposed a one-step direct registration method using multiple 2D slice stacks, termed as Slice Intersection Motion Correction (SIMC). The main idea of SIMC is based on an intuitive concept: The voxel values in all intersecting slice pairs acquired by orthogonally planned slice scheme should be the same if data are acquired in motion-free conditions. The mismatch is caused by motion. Thus, the amount of motion can be deduced by averaging the intensity differences across every voxel along an intersection, then across every possible intersection of a slice. SIMC is considered a variant of SVR with the same approach of multiple stacks for redundant sampling and rigid body assumption. SIMC fully utilizes 
the orthogonal property between stacks and it is more suitable for parallel computing.

Algorithms based on SVR and SIMR are typically very slow which hinders the use in real clinical environments. A framework using multiple GPUs implemented by Kainz et al. [4] dramatically shortens the computing time featured with optimized 2D to 3D registration, super- resolution with automatic outlier rejection and an optional intensity bias correction. It provides a fully automatic motion correction pipeline and its implementation is scalable for modern GPU- based infrastructures. The publicly available source code released by the authors has boosted its popularity in fetal MRI motion correction.

The recent breakthrough in machine learning opens a new way for slice to volume registration. With sufficient data of slices and their orientations for training, a deep learning based method can extract features in 2D slices and map 2D slices to a 3D volume. Miao et al. [5] implemented a deep Convolutional Neural Network (CNN) based regression model to directly estimate the transformation parameters. While this method was developed for general medical image registration, it can be seamlessly integrated into fetal MRI. More recently, Hou et al. [6] presented a deep CNN based model with respect to a learned canonical atlas coordinate system. The network specifically designed for fetal brain MRI maps each slice to a volumetric atlas. One advantage of machine learning based methods is quick inferences from trained networks, while most optimization-based methods usually need long time iterations to find the transform parameters for registration.

Other than slice to volume registration scheme, Patch to Volume Registration (PVR) [7] introduces a specific amount of redundant information that is exploited with parallelized patchwise optimization, super-resolution, and automatic outlier rejection. PVR does not rely on rigid body assumption and it can be parallelized for reasonable computing time on GPUs. In general, PVR does not provide superior registration performance in comparison with SVR-based methods, nor does it run faster in computing. The advantage of PVR is that it can register a large field of view with non-rigidly deformation without sacrificing registration accuracy and computing time. It can reconstruct a $3 \mathrm{D}$ volume of the whole fetal body, uterus, and placenta from 2D slices.

\section{Conflict of Interest}

No conflict of interest.

\section{Acknowledgement}

None.

\section{References}

1. S Jiang, H Xue, A Glover, M Rutherford, D Rueckert, et al. (2007) MRI of moving subjects using multislice Snapshot images with Volume Reconstruction (SVR): Application to fetal, neonatal, and adult brain studies. IEEE Trans. Med. Imaging 26(7): 967-980.

2. K Keraudren, M Kuklisova Murgasova, V Kyriakopoulou, C Malamateniou, M A Rutherford, et al. (2014) Automated fetal brain segmentation from 2D MRI slices for motion correction. Neuroimage 101: 633-643.

3. K Kim, P Habas, F Rousseau, O Glenn, A J Barkovich, et al. (2010) Intersection Based Motion Correction of Multi-Slice MRI for 3D in utero Fetal Brain Image Formation. IEEE Trans Med Imaging 29(1): 146-158.

4. B Kainz, M Steinberger, WWein, M Kuklisova Murgasova, C Malamateniou, et al. (2015) Fast Volume Reconstruction from Motion Corrupted Stacks of 2D Slices. IEEE Trans. Med. Imaging 34(9): 1901-1913.

5. S Miao, Z J Wang, R Liao (2016) A CNN Regression Approach for RealTime 2D/3D Registration. IEEE Trans. Med. Imaging 35(5): 1352-1363.

6. B Hou, B Khanal, A Alansary, S McDonagh, A Davidson, et al. (2018) 3-D Reconstruction in Canonical Co- Ordinate Space from Arbitrarily Oriented 2-D Images. IEEE Trans. Med. Imaging 37(8):1737-1750.

7. A Alansary, M Rajchl, S G McDonagh, M Murgasova, M Damodaram, et al. (2017) PVR: Patch- to-Volume Reconstruction for Large Area Motion Correction of Fetal MRI. IEEE Trans. Med. Imaging 36(10): 2031-2044. 